\title{
Number of tumor foci predicts prognosis in papillary thyroid cancer
}

\author{
Ning $\mathrm{Qu}^{\dagger}$, Ling Zhang ${ }^{\dagger}$, Qing-hai Ji*, Yong-xue Zhu, Zhuo-ying Wang, Qiang Shen, Yu Wang and Duan-shu Li
}

\begin{abstract}
Background: Papillary thyroid cancer (PTC) often presents as multifocal. However, the association of multifocality with poor prognosis remains controversial. The aim of this retrospective study was to identify the characteristics of PTC with multiple foci and to evaluate the association between multifocality and prognosis.

Methods: We reviewed the medical records of 496 patients who underwent total thyroidectomy for PTC. Patients were classified as G1 (1 tumor focus), G2 (2 foci), and G3 (3 or more foci). We analyzed the clinicopathological features and clinical outcomes in each classification. A Cox regression model was used to assess the relationship between multifocality and recurrence or cancer mortality.

Results: The G1, G2 and G3 groups included 287, 141 and 68 patients, respectively. The mean age was $47.1 \pm$ $16.1 \mathrm{yr}$ in G1, $41.1 \pm 18.4 \mathrm{yr}$ in $\mathrm{G} 2$, and $35.5 \pm 15.9 \mathrm{yr}$ in $\mathrm{G} 3$ and differed significantly among the 3 groups ( $p=0.001)$. The proportion of extrathyroidal extension, central lymph node metastasis (CLNM), and lateral lymph node metastasis (LLNM) in the G1 to G3 groups increased with increasing number of tumor foci. The Kaplan-Meier curves revealed that $\mathrm{G} 3$ had the shortest recurrence-free survival, and differences were significant among the 3 groups ( $p=0.001$, Log Rank test). Furthermore, cancer-specific survival rates decreased significantly with increasing number of tumor foci $(p=0.041)$. Independent predictors of recurrence by multivariate Cox analysis included $>3$ tumor foci [HR 2.60, 95\% confidence interval (Cl) 1.53-4.39, $p=0.001]$ and extrathyroidal extension (HR 1.95, Cl 1.12-3.38, $p=0.018)$.
\end{abstract}

Conclusion: An increase in the number of tumors is associated with a tendency toward more aggressive features and predicts poor prognosis in PTC.

Keywords: Papillary thyroid carcinoma, Multifocality, Recurrence, Mortality

\section{Background}

The number of newly diagnosed thyroid carcinoma cases is increasing annually, and an estimated 62,980 cases will be diagnosed in the United States in 2014, more than $90 \%$ of which will be papillary thyroid cancer (PTC) $[1,2]$. PTC often presents as multifocal tumors [3], which are thought to arise independently, indicating an inherent propensity to develop PTC, and spread throughout the thyroid gland [4].

PTC is generally an indolent disease and has a favorable prognosis in most affected patients; however, PTC with multifocal foci is likely to be aggressive and, accordingly,

\footnotetext{
* Correspondence: jiqinghai@shca.org.cn

${ }^{\dagger}$ Equal contributors

Department of Head and Neck Surgery, Fudan University Shanghai Cancer

Center; Department of Oncology, Shanghai Medical College, Fudan University, Shanghai 200032, China
}

require aggressive treatment [5,6]. The characteristics of PTC with multifocal foci and the prognostic significance of multifocality in PTC remain controversial $[7,8]$. We performed a retrospective analysis to examine the characteristics of multifocal PTC and the relationship between the number of tumor foci and PTC prognosis in a large group of Chinese patients with long-term follow-up.

\section{Methods \\ Patients}

The records of all PTC patients treated at our center between January 1, 1983, and December 31, 2007, were retrospectively reviewed. All patients provided written informed consent for their information to be stored in the hospital database and used for research, and this study was approved by the Ethical Committee of the Cancer

\section{Biomed Central}

(c) 2014 Qu et al.; licensee BioMed Central Ltd. This is an Open Access article distributed under the terms of the Creative Commons Attribution License (http://creativecommons.org/licenses/by/4.0), which permits unrestricted use, distribution, and reproduction in any medium, provided the original work is properly credited. The Creative Commons Public Domain Dedication waiver (http://creativecommons.org/publicdomain/zero/1.0/) applies to the data made available in this article, unless otherwise stated. 
Center of Fudan University. A series of 496 consecutive PTC patients who underwent primary surgical therapy of total thyroidectomy (TT) were enrolled in this study, representing $23.5 \%$ of the total 2115 patients treated at our center during the corresponding period.

\section{Initial treatment}

Before surgery, each patient underwent an ultrasonography (US) examination. US-guided fine-needle aspiration (FNA) was not performed routinely. Lobectomy plus ipsilateral central lymph node dissection (CLND) was typically performed as the initial surgical treatment for PTC patients with malignant lesions that were limited to a single lobe [9]. When the patient was older than $45 \mathrm{yr}$, the primary tumor was greater than $1 \mathrm{~cm}$, undetermined nodules were detected in the contralateral lobe by US or regional metastases or multifocal tumors were present, TT was performed at the time of the initial operation. When undetermined nodules were detected in the contralateral lobe by US without other factors, a subtotal lobectomy of approximately one-fourth to twothirds of the contralateral lobe was performed on the suspicion of lesions in the contralateral lobe following the preoperative US. Histology of the frozen sections (FS) assisted surgeons in determining the extent of the surgical procedures. When malignant lesions were identified in both lobes of the thyroid by FS after a subtotal lobectomy, a completion thyroidectomy (CT) was performed [9]. Modified lateral lymph node dissection (LLND), including levels II-V, was performed in cases with pathologically proven lymph node metastasis (LNM) or suspicious lymph nodes observed intraoperatively or on preoperative imaging.

Suppressive treatment with thyroid hormone was initiated after surgery to decrease serum thyroid-stimulating hormone (TSH) to subnormal levels without clinical thyrotoxicosis. Because its use is strictly controlled in China, radioactive iodine (RAI) therapy was not routinely prescribed for PTC patients after surgery unless patients had distant metastases [9].

\section{Clinicopathological variable assessment and statistical analysis}

Multifocality was defined as 2 or more tumor foci within the thyroid. For multifocal tumors, the diameter of the largest tumor focus was taken as the primary tumor. In addition, data on patient clinical features (gender, age at diagnosis), tumor histological characteristics (maximum size of tumor, extrathyroidal extension), and cervical LNM were extracted from the database.

\section{Follow-up}

All patients gave consent to participate in the follow-up study. The follow-up period for each patient was defined as the length of time from the initial therapy until the last known date of contact documented by a review of the medical record or as a follow-up phone call to the patient. Postsurgical physical examinations were performed every 3-6 months. During the follow-up visits, all patients underwent US examinations of the neck. Recurrence was defined as the appearance of disease, including new biopsy-proven/secondary surgery-confirmed local disease or distant disease revealed by ultrasonography and/or imaging scans, in any patient who had been free of disease (i.e., no palpable disease and negative radioactive iodine scan). New biopsy-proven/secondary surgery-confirmed local disease within the residual thyroid gland or lateral lymph nodes was classified as neck recurrence; distant disease revealed by ultrasonography and/or imaging scans of other sites, including the lungs, bones or brain, was classified as distant recurrence.

\section{Statistical analysis}

The results are expressed as the mean \pm standard deviation. Statistical analysis was performed using Student's $t$ test, $\mathrm{X}^{2}$ test or Mann-Whitney test as appropriate. Patients who were alive and who did not relapse were censored at the date of their last follow-up visit. Neck/metastasis recurrence-free survival (RFS) was defined as the time between the date of initial surgery and the first event of recurrence or death. Overall survival (OS) was defined as the time between the date of initial surgery and death (all causes or cancer-specific). Cancer-specific survival (CSS) was defined as the time between the date of initial surgery and cancer-specific death. Survival rates were estimated using the Kaplan-Meier method [10]. The hazard ratio (HR) and the 95\% confidence interval (CI) for relationships between each variable and recurrence were calculated using a binary Cox regression model [11]. A $p<0.05$ was considered statistically significant. Statistical analyses were performed using SPSS for Windows 13.0 (SPSS Inc., Chicago, IL).

\section{Results}

\section{Baseline characteristics of patients}

At the time of diagnosis, the 496 patients ranged in age from 7 to $85 \mathrm{yr}$ (mean $43.8 \pm 17.3 \mathrm{yr}$ ). The series comprised 336 females (67.7\%) and 160 males (32.3\%), with a female/male ratio of $2.1 / 1$. Based on postoperative pathology, the mean maximal tumor size was $2.31 \pm$ $1.59 \mathrm{~cm}$. Multifocality was observed in 209 patients (42.1\%). Extrathyroidal extension was evident in 207 cases $(41.7 \%)$. A total of 381 patients $(76.8 \%)$ exhibited central lymph node metastasis (CLNM), and 306 patients (61.7\%) exhibited lateral lymph node metastasis (LLNM). No patients had a history of familial PTC. The characteristics of the patients are presented in Table 1. 
Table 1 Clinicopathological characteristics of 496 papillary thyroid cancer patients

\begin{tabular}{lc}
\hline Variables & Number (\%) \\
\hline Male gender & $160(32.3)$ \\
Age & $43.8 \pm 17.3 \mathrm{yr}$ \\
Maximum size of tumor $(\mathrm{cm})$ & $2.31 \pm 1.59$ \\
Multifocality & $209(42.1)$ \\
Number of tumor foci & $1.2 \pm 1.3$ \\
Extrathyroidal extension & $207(41.7)$ \\
Central lymph node metastasis & $381(76.8)$ \\
Lateral lymph node metastasis & $306(61.7)$ \\
\hline
\end{tabular}

Data are presented as $\mathrm{n}(\%)$ or mean \pm standard deviation.

\section{Comparison among groups according to the number of tumor foci}

Based on the number of tumor foci, the population was divided into 3 groups: G1 (1 tumor focus), G2 (2 foci), and G3 (3 or more foci). Patients with a solitary tumor $(\mathrm{n}=287,57.9 \%)$ were classified as G1. Patients with multifocal tumors were classified as $\mathrm{G} 2(\mathrm{n}=141,28.4 \%)$ or G3 ( $n=68,13.7 \%)$. Table 2 presents the characteristics of patients according to the number of tumor foci.

The mean age was $47.1 \pm 16.1 \mathrm{yr}$ in G1, 41.1 $\pm 18.4 \mathrm{yr}$ in $\mathrm{G} 2$, and $35.5 \pm 15.9 \mathrm{yr}$ in G3 and differed significantly among the 3 groups $(p=0.001)$. A decreasing trend of age was observed from G1 to G3 according to the increasing number of tumor foci.

The incidence of neck recurrence was highest in group G3, followed by group G2 and group G1, and differed

Table 2 Clinicopathological features of papillary thyroid cancer patients according to the number of tumor Foci

\begin{tabular}{|c|c|c|c|c|}
\hline Variables & $\begin{array}{c}\mathrm{G} 1 \\
(\mathrm{n}=287)\end{array}$ & $\begin{array}{c}G 2 \\
(n=141)\end{array}$ & $\begin{array}{c}\text { G3 } \\
(n=68)\end{array}$ & $p$ value \\
\hline Male gender & $94(32.8)$ & $43(30.5)$ & $23(33.8)$ & NS \\
\hline Age & $47.1 \pm 16.1 \mathrm{yr}$ & $41.1 \pm 18.4 \mathrm{yr}$ & $35.5 \pm 15.9 \mathrm{yr}$ & $<0.05$ \\
\hline $\begin{array}{l}\text { Maximum size } \\
\text { of tumor }(\mathrm{cm})\end{array}$ & $2.29 \pm 1.41$ & $2.35 \pm 1.9$ & $2.37 \pm 1.60$ & NS \\
\hline $\begin{array}{l}\text { Extrathyroidal } \\
\text { extension }\end{array}$ & $115(40.1)$ & $60(42.6)$ & $32(47.1)$ & NS \\
\hline $\begin{array}{l}\text { Central lymph } \\
\text { node metastasis }\end{array}$ & $215(74.9)$ & $110(78.0)$ & $56(82.4)$ & NS \\
\hline $\begin{array}{l}\text { Lateral lymph } \\
\text { node metastasis }\end{array}$ & $173(60.3)$ & $80(56.7)$ & $53(77.9)$ & NS \\
\hline Neck recurrence & $19(6.6)$ & $24(17.6)$ & $14(20.6)$ & $<0.05$ \\
\hline Distant recurrence & $8(2.8)$ & $7(5.0)$ & $7(10.3)$ & NS \\
\hline $\begin{array}{l}\text { Died of thyroid } \\
\text { cancer }\end{array}$ & $4(1.4)$ & $6(6.4)$ & $5(7.4)$ & NS \\
\hline
\end{tabular}

Data are presented as $\mathrm{n}(\%)$ or mean \pm standard deviation.

Abbreviations: G1 1 tumor focus, G2 2 tumor foci, G3 3 or more tumor foci, NS not significant.

Age at diagnosis and primary tumor size were compared using the Kruskal-Wallis test.

Group comparisons of categorical variables were performed using the chi-square test or, for small cell values, Fisher's exact test. significantly among the 3 groups $(p=0.037)$. However, there were no significant differences with respect to gender, maximum tumor size, extrathyroidal extension, CLNM, LLNM, distant recurrence or cancer-specific death among the 3 groups. However, the proportion of aggressive features, such as extrathyroidal extension, CLNM, and LLNM, in the G1 to G3 groups exhibited an increasing trend according to the number of tumor foci.

\section{Number of tumor foci and recurrence}

The mean length of follow-up was $124.3 \pm 67.8$ months, with a range of 10 to 343 months. During the follow-up period, 57 patients (11.5\%) experienced neck recurrence, including recurrence in the thyroid or operation bed $(n=13)$, lateral neck $(n=40)$, or both locations $(n=4)$. Distant recurrences occurred in 22 patients (4.4\%), including 16 lung metastases, 5 bone metastases, and 1 brain metastasis. Four patients exhibited both neck recurrence and distant recurrence; therefore, the frequency of total recurrence was $15.1 \%$ (75/496), and the RFS rate was $84.3 \%$ at $10 \mathrm{yr}, 70.8 \%$ at $15 \mathrm{yr}$, and $69.6 \%$ at $20 \mathrm{yr}$ from the time of the initial operation. The Kaplan-Meier curve was used to investigate the differences in RFS rates among the 3 groups (Figure 1). Patients with 3 or more tumor foci exhibited the shortest RFS, followed by G1 and G2, and differences were significant among the 3 groups $(p=0.001$, Log Rank test).

To determine how strongly the number of tumor foci was associated with recurrence relative to other known predictors of recurrence in PTC, we performed multivariate Cox regression analysis. Instead of limiting the multivariate analysis to the significant terms from the univariate analysis, we included all variables because these factors have been previously demonstrated to be important in predicting disease recurrence in adult PTC patients. The results are presented in Table 3. The risk of recurrence increased with increasing number of tumor foci, and G3 exhibited a greater risk of recurrence than

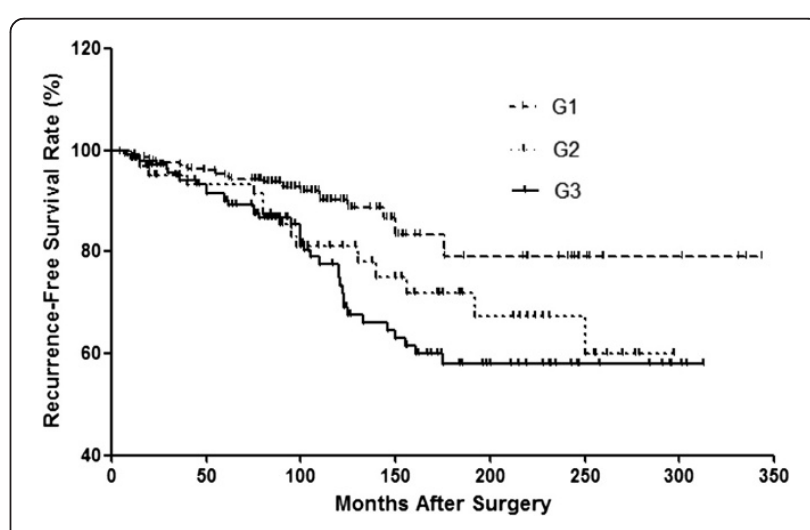

Figure 1 Kaplan-Meier curves for recurrence-free survival (RFS) in the G1, G2 and G3 groups. (Log Rank test, $p=0.001$ ). 
Table 3 Multivariate cox regression for recurrence

\begin{tabular}{lcc}
\hline Independent variable & \multicolumn{2}{c}{ Multivariate analysis } \\
\cline { 2 - 3 } & HR (95\% Cl) & $\boldsymbol{p}$ value \\
\hline Gender (female vs. male) & $0.65(0.41-1.03)$ & 0.063 \\
Age & $1.00(0.99-1.02)$ & 0.872 \\
Size & $1.12(0.98-1.28)$ & 0.108 \\
Extrathyroidal extension & $1.95(1.12-3.38)$ & 0.018 \\
Number of tumor foci & & \\
G1 (1 tumor focus) & 1 (reference) & \\
G2 (2 foci) & $2.06(1.05-4.01)$ & 0.034 \\
G3 (3 or more foci) & $2.60(1.53-4.39)$ & 0.001 \\
Central lymph node metastasis & $0.65(0.33-1.25)$ & 0.194 \\
Lateral lymph node metastasis & $1.07(0.52-2.16)$ & 0.862 \\
\hline
\end{tabular}

the G1 group (HR 2.60, 95\% CI 1.53-4.39, $p=0.001$ ). In addition, extrathyroidal extension was an independent predictor of recurrence (HR 1.95, 95\% CI $1.12-3.38$, $p=0.018)$.

\section{Number of tumor foci and survival}

Deaths were observed in 45 patients; however, cancerspecific deaths only occurred in 15 patients. OS and CSS were $92.4 \%$ and $96.9 \%$ at $10 \mathrm{yr}, 86.7 \%$ and $94.6 \%$ at $15 \mathrm{yr}$, and $78.6 \%$ and $89.1 \%$ at $20 \mathrm{yr}$ from the initial operation, respectively. The Kaplan-Meier curve was used to investigate the differences in CSS rates among the 3 groups (Figure 2). The G3 group exhibited the lowest rate of CSS; thus, PTC patients with 3 or more tumor foci had the greatest risk of recurrence, followed by group G2 and group G1. Although a trend of decreasing CSS with increasing number of tumor foci was observed, the differences in CSS were not significant among the 3 groups $(p=0.087$, Log Rank test).

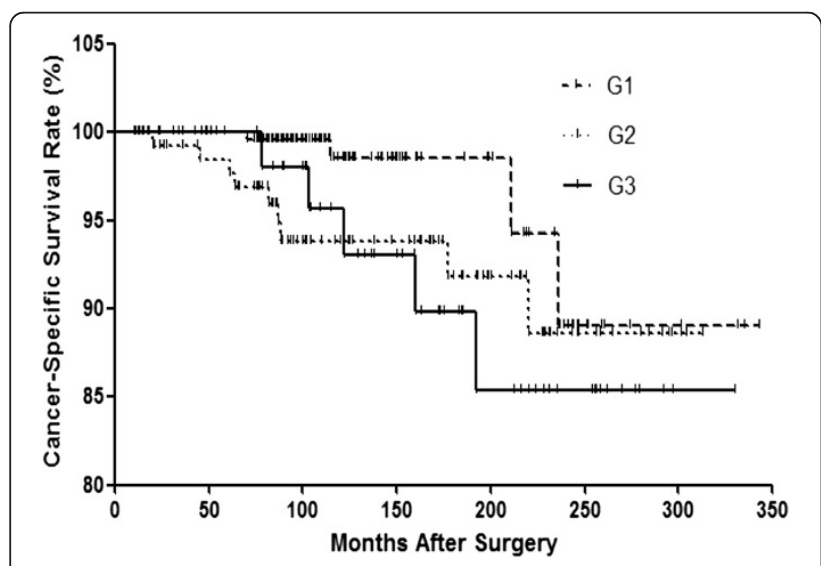

Figure 2 Kaplan-Meier curves for cancer-specific survival (CSS) in the G1, G2 and G3 groups. (Log Rank test, $p=0.087$ ).

\section{Complications}

Of the 496 patients, 26 (5.2\%) had transient hypoparathyroidism, and 6 (1.2\%) had permanent hypoparathyroidism. Recurrent laryngeal nerve injury occurred in 11 patients $(2.2 \%)$, of which 2 were transient $(0.4 \%)$ and 9 were permanent $(1.8 \%)$.

\section{Discussion}

In this study, we investigated the relationship between the number of tumor foci and other clinicopathological features and the impact of multifocality on prognosis in PTC. We observed that an increasing number of tumor foci was associated with both a tendency toward more aggressive features as well as poor prognosis in PTC.

Few studies have addressed the associations between the number of tumor foci and clinicopathological features in multifocal PTCs. Kim et al. [12] reported that an increase in the number of tumor foci was strongly associated with older age at diagnosis, cervical LNM, and advanced TNM stage of PTC; furthermore, the number of tumor foci independently predicted LNM. In accordance with their findings, we observed that an increasing number of tumor foci was associated with a tendency toward more aggressive features, such as larger primary tumor size, more frequent extrathyroidal extension and cervical LNM. Although these differences were too small to be significant, they suggest that multifocality might represent the tumor burden and predict more aggressive behavior during disease progression. An interesting finding of the current study was that an increasing number of tumor foci was associated with a younger age at diagnosis in PTC, in contrast to previous reports that advanced age is a prognostic factor for poor prognosis in PTC [12-15]. Younger age may indicate greater risk for the evolution of biological aggressiveness, such as multifocality; we previously reported that younger age is significantly associated with cervical LNM in PTC. However, appropriate initial management may improve the prognosis of younger PTC patients. Younger age as a predictor of multifocality emphasizes the need for more aggressive therapy to achieve the relatively preferable outcomes observed in elderly patients.

Recent studies of the relationship between multifocality and oncological outcomes have suggested that multifocal lesions in PTC are positively associated with poorer prognosis in patients. Kim et al. [16] reviewed the medical records of 2095 patients who underwent total thyroidectomy for PTC and reported that multifocality is associated with an increased risk of disease recurrence and persistence, suggesting that the number of tumor foci is a significant predictor of poor clinical outcomes. We determined that multifocality is a risk factor for poor outcomes; furthermore, we observed that a higher number of tumors had a strong linear effect on the risk of recurrence (Table 2 and Figure 1) and was also associated with a trend toward a 
higher probability for cancer mortality (Table 2), whereas CSS rates did not differ significantly among the 3 groups (Figure 2). In addition, the predictive impact of tumor numbers on recurrence was persistent in multivariate Cox regression analysis when all variables were included (Table 3). Thus, a higher number of tumors, particularly $\geq 3$ tumor foci, is an independent predictor of disease recurrence and a possible indicator of cancer death in PTC. Extrathyroidal extension was another predictor for recurrence, consistent with previous studies [17-19]. However, some studies of the prognostic impact of tumor multifocality in papillary thyroid microcarcinoma (PTMC) have suggested that multifocal lesions do not appear to have prognostic impact in PTMC [20]. Given the high incidence of multifocality in PTMC, $20-40 \%$ [21-23], further studies of small PTC tumors are needed to investigate the true role and influence of multifocality in this subtype of PTC.

At our center, TT was not routinely performed in PTC as an initial treatment. Considering the high incidence and negative influence of multifocality, extensive TT surgery is more likely to remove all disease foci and improve prognosis, particularly in PTC patients with $\geq 3$ tumor foci and extrathyroidal extension. In addition, the rates of complications for permanent hypoparathyroidism and recurrent laryngeal nerve injury were both within the ranges of $0-4 \%$ and $0-6 \%$ reported in previous studies $[24,25]$, suggesting that thyroidectomy plus lymph node dissections can be performed safely and with low morbidity by experts.

Our study has several limitations that must be taken into account. First, since this study was a retrospective analysis, the prognostic significance of tumor foci has not been fully investigated. The long-term follow-up studies are needed to confirm the prognostic significance in PTC. Second, our study population was a cohort of patients cared for in a single center. Therefore, a much larger number of subjects in multicenter will be needed to generalize this results. At last, the fact that patients in China only receive radioiodine for distant metastases may significantly alter the outcome of patients in other countries where radioiodine is given, but this study certainly shows the impact of significant sized multifocality when radioiodine is not given.

\section{Conclusion}

We observed that a higher number of PTC foci was associated with a tendency toward more aggressive features, including greater primary tumor size, more frequent extrathyroidal extension and cervical LNM. In addition, an increase in the number of tumors was associated with an increased risk of recurrence and a trend toward more cancer mortality. Our findings suggested that the number of tumor foci could be used to assess the risk of poor prognosis, and TT is recommended in patients with more tumor foci.

\section{Abbreviations}

PTC: Papillary thyroid cancer; LNM: Lymph node metastasis; CLNM: Central lymph node metastasis; LLNM: Lateral lymph node metastasis; TT: Total thyroidectomy; CLND: Central lymph node dissection; LLND: Lateral lymph node dissection; CSS: Cancer-specific survival; RFS: Recurrence-free survival; US: Ultrasonography; FNA: Fine-needle aspiration; FS: Frozen sections;

TSH: Thyroid-stimulating hormone; RAl: Radioactive iodine.

\section{Competing interests}

The authors declared that they have no competing interests.

\section{Authors' contributions}

Q-hJ, Y-xZ, and D-sL designed the study. Q-hJ, Z-yW, and QS provided the databases. NQ, LZ, and YW assembled and analyzed the data. NQ and LZ drafted the article, and Q-hJ and Y-xZ critically revised the article for important intellectual content. Q-hJ gave final approval of the version to be published. All authors read and approved the final manuscript.

\section{Acknowledgments}

The funders had no role in study design, data collection and analysis, decision to publish, or preparation of the manuscript. Address all correspondence and requests for reprints to Professor Qing-hai Ji, Department of Head and Neck Surgery, Fudan University Cancer Hospital, Department of Oncology, Shanghai Medical College, 270 Dong An Road, Shanghai 200032, People's Republic of China. E-mail: jiqinghai@shca.org.cn.

\section{Source(s) of funding}

This research is supported by grants from the National Natural Science Foundation of China (81272934) and the Natural Science Foundation of Shanghai (12ZR1406800)

Received: 14 July 2014 Accepted: 26 November 2014

Published: 4 December 2014

\section{References}

1. Siegel R, Ma J, Zou Z, Jemal A: Cancer statistics, 2014. CA Cancer J Clin 2014, 64(1):9-29.

2. Hung W, Sarlis NJ: Current controversies in the management of pediatric patients with well-differentiated nonmedullary thyroid cancer: a review. Thyroid 2002, 12(8):683-702.

3. Katoh R, Sasaki J, Kurihara H, Suzuki K, lida Y, Kawaoi A: Multiple thyroid involvement (intraglandular metastasis) in papillary thyroid carcinoma. A clinicopathologic study of 105 consecutive patients. Cancer 1992, 70(6):1585-1590

4. Jovanovic L, Delahunt B, Mclver B, Eberhardt NL, Bhattacharya A, Lea R, Grebe SK: Distinct genetic changes characterise multifocality and diverse histological subtypes in papillary thyroid carcinoma. Pathology 2010, 42(6):524-533.

5. Baek SK, Jung KY, Kang SM, Kwon SY, Woo JS, Cho SH, Chung EJ: Clinical risk factors associated with cervical lymph node recurrence in papillary thyroid carcinoma. Thyroid 2010, 20(2):147-152.

6. Leboulleux S, Rubino C, Baudin E, Caillou B, Hartl DM, Bidart JM, Travagli JP, Schlumberger M: Prognostic factors for persistent or recurrent disease of papillary thyroid carcinoma with neck lymph node metastases and/or tumor extension beyond the thyroid capsule at initial diagnosis. J Clin Endocrinol Metab 2005, 90(10):5723-5729.

7. Mazzaferri EL, Jhiang SM: Long-term impact of initial surgical and medical therapy on papillary and follicular thyroid cancer. Am J Med 1994, 97(5):418-428.

8. Chow SM, Law SC, Chan JK, Au SK, Yau S, Lau WH: Papillary microcarcinoma of the thyroid-Prognostic significance of lymph node metastasis and multifocality. Cancer 2003, 98(1):31-40.

9. Zhang L, Wei WJ, Ji QH, Zhu YX, Wang ZY, Wang Y, Huang CP, Shen Q, Li DS, Wu Y: Risk factors for neck nodal metastasis in papillary thyroid microcarcinoma: a study of 1066 patients. J Clin Endocrinol Metab 2012, 97(4):1250-1257.

10. Dinse GE, Lagakos SW: Nonparametric estimation of lifetime and disease onset distributions from incomplete observations. Biometrics 1982, 38(4):921-932

11. Gill RD: Multistate life-tables and regression models. Math Popul Stud 1992, 3(4):259-276. 
12. Kim HJ, Park HK, Byun DW, Suh K, Yoo MH, Min YK, Kim SW, Chung JH: Number of tumor foci as predictor of lateral lymph node metastasis in papillary thyroid carcinoma. Head Neck 2014, doi:10.1002/hed.23650.

13. Zhao Q, Ming J, Liu C, Shi L, Xu X, Nie X, Huang T: Multifocality and total tumor diameter predict central neck lymph node metastases in papillary thyroid microcarcinoma. Ann Surg Oncol 2013, 20(3):746-752.

14. Zhou YL, Gao EL, Zhang W, Yang H, Guo GL, Zhang XH, Wang OC: Factors predictive of papillary thyroid micro-carcinoma with bilateral involvement and central lymph node metastasis: a retrospective study. World I Surg Oncol 2012, 10:67.

15. Shepet K, Alhefdhi A, Lai N, Mazeh H, Sippel R, Chen H: Hereditary medullary thyroid cancer: age-appropriate thyroidectomy improves disease-free survival. Ann Surg Oncol 2013, 20(5):1451-1455.

16. Kim HJ, Sohn SY, Jang HW, Kim SW, Chung JH: Multifocality, but not bilaterality, is a predictor of disease recurrence/persistence of papillary thyroid carcinoma. World J Surg 2013, 37(2):376-384.

17. Edge SB, Compton CC: The American Joint Committee on Cancer: the 7th edition of the AJCC cancer staging manual and the future of TNM. Ann Surg Oncol 2010, 17(6):1471-1474.

18. Ito Y, Tomoda C, Uruno T, Takamura Y, Miya A, Kobayashi K, Matsuzuka F, Kuma K, Miyauchi A: Clinical significance of metastasis to the central compartment from papillary microcarcinoma of the thyroid. World J Surg 2006, 30(1):91-99.

19. Wada N, Nakayama H, Suganuma N, Masudo Y, Rino Y, Masuda M, Imada T: Prognostic value of the sixth edition AJCC/UICC TNM classification for differentiated thyroid carcinoma with extrathyroid extension. J Clin Endocrinol Metab 2007, 92(1):215-218.

20. Riss JC, Peyrottes I, Chamorey E, Haudebourg J, Sudaka A, Benisvy D, Marcy PY, Nao EE, Demard F, Vallicioni J, Poissonnet G, Dassonville O, Santini J, Bozec A: Prognostic impact of tumour multifocality in thyroid papillary microcarcinoma based on a series of 160 cases. Eur Ann Otorhinolaryngol Head Neck Dis 2012, 129(4):175-178.

21. Ogilvie JB, Patel KN, Heller KS: Impact of the 2009 American Thyroid Association guidelines on the choice of operation for well-differentiated thyroid microcarcinomas. Surgery 2010, 148(6):1222-1226. discussion 1226-1227.

22. Mercante G, Frasoldati A, Pedroni C, Formisano D, Renna L, Piana S, Gardini G, Valcavi R, Barbieri V: Prognostic factors affecting neck lymph node recurrence and distant metastasis in papillary microcarcinoma of the thyroid: results of a study in 445 patients. Thyroid 2009, 19(7):707-716.

23. Connor MP, Wells D, Schmalbach CE: Variables predictive of bilateral occult papillary microcarcinoma following total thyroidectomy. Otolaryngol Head Neck Surg 2011, 144(2):210-215.

24. Moo TA, Umunna B, Kato M, Butriago D, Kundel A, Lee JA, Zarnegar R, Fahey TJ 3rd: Ipsilateral versus bilateral central neck lymph node dissection in papillary thyroid carcinoma. Ann Surg 2009, 250(3):403-408,

25. Koo BS, Choi EC, Yoon YH, Kim DH, Kim EH, Lim YC: Predictive factors for ipsilateral or contralateral central lymph node metastasis in unilateral papillary thyroid carcinoma. Ann Surg 2009, 249(5):840-844.

doi:10.1186/1471-2407-14-914

Cite this article as: Qu et al:: Number of tumor foci predicts prognosis in papillary thyroid cancer. BMC Cancer 2014 14:914.

\section{Submit your next manuscript to BioMed Central and take full advantage of:}

- Convenient online submission

- Thorough peer review

- No space constraints or color figure charges

- Immediate publication on acceptance

- Inclusion in PubMed, CAS, Scopus and Google Scholar

- Research which is freely available for redistribution

Submit your manuscript at www.biomedcentral.com/submit
C Biomed Central 